\title{
Trastornos del aprendizaje y epilepsia
}

\author{
Transtornos de aprendizagem e epilepsia \\ Learning disorder and epilepsy
}

\author{
Fernando César de Medeiros Rocha Junior \\ ORCID: https://orcid.org/0000-0002-2976-4640 \\ Universidad Politécnica y Artística del Paraguay, Paraguay \\ E-mail: fernandocmrj@gmail.com \\ Glauber José Alencar Pereira \\ ORCID: https://orcid.org/0000-0002-5729-8368 \\ Universidad Politécnica y Artística del Paraguay, Paraguay \\ E-mail: glauberjap@hotmail.com \\ Hyorrana Rayanne de Oliveira Santos \\ ORCID: https://orcid.org/0000-0002-0027-0460 \\ Universidad Politécnica y Artística del Paraguay, Paraguay \\ E-mail: hyorranaros@gmail.com \\ Renata Manoela Rocha de Oliveira \\ ORCID: https://orcid.org/0000-0002-0614-7121 \\ Universidad Politécnica y Artística del Paraguay, Paraguay \\ E-mail: renatadouca@hotmail.com \\ Maria de los Angeles Pedroso Rodriguez \\ ORCID: https://orcid.org/0000-0001-8745-5144 \\ Universidad Politécnica y Artística del Paraguay, Paraguay \\ E-mail: mariaapedroso@yahoo.com \\ Seidel Guerra López \\ ORCID: https://orcid.org/0000-0001-7251-3911 \\ Universidad Politécnica y Artística del Paraguay, Paraguay \\ E-mail: seidelguerra@yahoo.es
}

\begin{abstract}
Resumen
La epilepsia es un trastorno cerebral caracterizado por una predisposición continuada a la aparición de crisis epilépticas y por las consecuencias neurobiológicas, cognitivas, psicológicas y sociales de esta enfermedad. La definición de epilepsia requiere la presencia de al menos una crisis epiléptica. Los niños con epilepsia tienen mayor riesgo de tener trastornos del aprendizaje en comparación con los niños sanos y con aquellos que presentan otras enfermedades crónicas de la infancia. Se realizo una revisión narrativa de la literatura, incluyendo artículos indexados en las plataformas digitales, Literatura Latino-Americana e do Caribe em Ciencias da Saúde (LILACS), Health Information from the National Library of Medicine (Medline), Web of Science, en la biblioteca electrónica Scientific Eletronic Library On-line (SciELO), y PubMed, publicados en el periodo comprendido entre los años 2017 y 2020. Los trastornos de aprendizaje son más frecuentes entre los niños con epilepsia, con un predomino significativo en el sexo masculino; la Epilepsia Rolándica es la que presenta mayor asociación con los trastornos del aprendizaje; el inicio más temprano de las convulsiones está relacionado con mayor deterioro cognitivo y existe una alta comorbilidad entre la epilepsia, los Trastornos por Déficit de Atención e Hiperactividad y los trastornos generalizados del aprendizaje.
\end{abstract}

Palabras clave: Epilepsia; Trastornos de aprendizaje; niños.

\section{Resumo}

A epilepsia é uma doença cerebral caracterizada por uma predisposição contínua ao aparecimento de crises epilépticas e pelas consequências neurobiológicas, cognitivas, psicológicas e sociais desta doença. A definição de epilepsia requer a presença de pelo menos uma crise epiléptica. Crianças com epilepsia correm maior risco de dificuldades de aprendizagem em comparação com crianças saudáveis e com outras doenças crônicas da infância. Foi realizada uma revisão narrativa da literatura, incluindo artigos indexados em plataformas digitais, Literatura Latino-Americana e do Caribe em Ciências da Saúde (LILACS), Informações em Saúde da Biblioteca Nacional de Medicina (Medline), Web of Science, na biblioteca eletrônica Scientific Eletronic Library On-line (SciELO), e PubMed, publicada no período de 2017 a 2020. Os distúrbios de aprendizagem são mais frequentes nas crianças com epilepsia, com predomínio significativo no sexo masculino; A epilepsia rolândica é a que apresenta maior associação com distúrbios de aprendizagem; o início precoce das crises está relacionado a um maior comprometimento cognitivo e há uma alta 
comorbidade entre epilepsia, transtornos de déficit de atenção e hiperatividade e transtornos generalizados de aprendizagem.

Palavras-chave: Epilepsia; Distúrbios de aprendizagem; Crianças.

\begin{abstract}
Epilepsy is a brain disorder characterized by a continuous predisposition to the appearance of epileptic seizures and by the neurobiological, cognitive, psychological and social consequences of this disease. The definition of epilepsy requires the presence of at least one epileptic seizure. Children with epilepsy are at higher risk for learning disabilities compared to healthy children and those with other chronic childhood diseases. A narrative review of the literature was carried out, including articles indexed in digital platforms, Literatura Latino-Americana e do Caribe em Ciencias da Saúde (LILACS), Health Information from the National Library of Medicine (Medline), Web of Science, in the electronic library Scientific Eletronic Library On-line (SciELO), and PubMed, published in the period between 2017 and 2020. Learning disorders are more frequent among children with epilepsy, with a significant predominance in males; Rolandic Epilepsy is the one with the greatest association with learning disorders; the earlier onset of seizures is related to greater cognitive impairment and there is a high comorbidity between epilepsy, Attention Deficit and Hyperactivity Disorders and generalized learning disorders.
\end{abstract}

Keywords: Epilepsy; Learning disorders; Children.

\title{
1. Introducción
}

La epilepsia se define como una enfermedad crónica del cerebro caracterizada por una predisposición duradera a generar convulsiones, no provocadas por ninguna agresión inmediata del sistema nervioso central, y por las consecuencias neurobiológicas, cognitivas, psicológicas y sociales de las recurrencias de estas convulsiones (Beghi, 2020), que en 50\% de los casos sucede en la infancia (Pavlou \& Gkampeta, 2011). Mientras todas las personas con epilepsia experimentan crises epileptogénicas, no todas las personas con convulsiones tienen epilepsia (Beghi, 2020)

La prevalencia de la epilepsia difiere significativamente entre países, esto puede explicarse por la utilización de diferente abordaje metodológico, factores genéticos (Guillén-Pinto et al., 2019) y por la mayor frecuencia de algunos factores de riesgos, como: problemas perinatales, tasas más altas de infecciones del sistema nervioso central y lesión cerebral traumática en los países de ingresos bajos y medianos (Beghi, 2020). A lo largo de la vida, la prevalencia de esta enfermedad es de 7,60 por 1000 habitantes, pero es mayor en los países en vías de desarrollo, con resultado de 8,75 por 1000 (Conde Pérez et al., 2013; Fiest et al., 2017).

Pacientes en edades extremas de la vida (niños y ancianos) son los más afectados por la epilepsia (Fiest et al., 2017). Camfield and Camfield en el año 2015 estimaron que 86 por cada 100.000 habitantes en una población bien definida durante el primer año de edad padecen epilepsia, con una tendencia a disminuir alrededor de 23-31 por 100.000 en personas con edades entre 30 y 59 años, y un aumento posterior de hasta 180 por 100.000 en el grupo de personas con más de 85 años (Beghi, 2020). En los niños, la incidencia de epilepsia es más alta en el primer año de vida y desciende hasta los niveles del adulto al final de los 10 años de edad (Camfield \& Camfield, 2015).

De todos los casos de epilepsia infantil (entre 5 y 14 años), la Epilepsia Rolándica Centro Temporal (Epilepsia con Puntas Benignas de la Infancia) es responsable del 8 al $25 \%$ de los casos. Generalmente, el inicio de la enfermedad se produce entre 3 y 13 años y se resuelve espontáneamente en la edad adulta (Smith et al., 2015).

A lo largo del tiempo, la epilepsia se ha asociado con dificultades académicas y socioeconómicas de los pacientes (Pavlou \& Gkampeta, 2011). Los individuos en edades escolares que padecen de epilepsia son más propensos a tener dificultades con el aprendizaje, problemas cognitivos, sociales, conductuales y emocionales, en comparación con los niños sanos. Los pacientes pediátricos con epilepsia presentan frecuentemente trastornos atencionales, que afectan primordialmente los mecanismos de atención sostenida, sí además presentan descargas epileptogénicas durante el sueño, se afecta también la atención selectiva o dividida (Martinez, 2018). 
Se entiende por aprendizaje a los cambios comportamentales influenciados por la plasticidad neural cognitiva. Alteraciones en este proceso llevan a los trastornos del aprendizaje, que promueven disturbios psicomotores, motores, en la atención, desinterés, memorización y problemas comportamentales de los pacientes. A consecuencia del disturbio en la atención, los pacientes tienen una inadecuada conexión entre la percepción y el procesamiento de las informaciones (Gomes et al., 2013).

De todos los síndromes epilépticos, la epilepsia Rolándica es la que se asocia principalmente con los Trastornos del Aprendizaje (TA), y los niños con este tipo de epilepsia se desempeñaron significativamente peor que los controles en: ortografía, lectura en voz alta y comprensión de lectura, y con frecuencia tuvieron un desempeño escolar por debajo del promedio (Martinez, 2018)

Existe una elevada comorbilidad neuro-conductual en los niños epilépticos, que puede llegar hasta un $80 \%$ de los pacientes (Reilly et al., 2014). El Trastorno por Déficit de Atención e Hiperactividad (TDAH) se reportó en un 33\%, la discapacidad intelectual en $40 \%$ y el trastorno del espectro autista en $21 \%$ de los casos. En comparación con niños sanos, los pacientes con epilepsia activa, son más diagnosticados con depresión, ansiedad, trastornos de conducta y deficiencia mental (Russ et al., 2012).

Los Trastornos del aprendizaje son definidos como trastornos que interfieren significativamente con el rendimiento académico o con las actividades diarias que requieren habilidades de lectura, escritura o matemáticas en sujetos con un Cociente Intelectual (CI) normal y son considerados uno de los factores más importantes que conducen a un resultado académico deficiente (Pavlou \& Gkampeta, 2011). Por otra parte, el problema se dificulta porque algunas escuelas actualmente no ofrecen seguridad académica y transdisciplinariedad a los niños con este trastorno (Patricia et al., 2018). Así, la prevalencia de los TA parece haber aumentado en las últimas décadas y esto puede estar relacionado con la mejoría en las tasas de alfabetización y la mayor atención prestada al problema (Pavlou \& Gkampeta, 2011).

Cuando la epilepsia se aparece en la primera infancia, los patrones de desarrollo de las áreas corticales del lenguaje en el cerebro pueden afectarse y aparecer alteraciones del neurodesarrollo cortical, por tanto, el niño puede tener dificultades en la adquisición de habilidades de comunicación. Esta perturbación puede extenderse, eventualmente, a los efectos sobre la lectura y la escritura (Busto, 2018). La mayoría de los niños con TA tienen déficits primarios en discapacidades básicas de lectura (Pavlou \& Gkampeta, 2011).

Se han asociado múltiples factores con los déficits de aprendizaje en los pacientes con epilepsia, como factores psicosociales, medicamentosos y relacionados propiamente con la neurobiología de la epilepsia (Pavlou \& Gkampeta, 2011) Los fármacos antiepilépticos pueden tener efectos adversos sobre el comportamiento, la arquitectura normal del sueño y la cognición, y agravar así la repercusión que tiene la propia epilepsia sobre el desarrollo y el aprendizaje del niño (Ron, 2017)

Un inicio más temprano de las convulsiones, alrededor de 2 años, está relacionado con un mayor deterioro cognitivo. Las posibles razones de esto incluyen: el daño al cerebro causado por las convulsiones tempranamente interfiere con el desarrollo de las funciones de aprendizaje, la aparición temprana puede aumentar la vulnerabilidad del cerebro al efecto deletéreo de las convulsiones y por otra parte, es más probable que las convulsiones de inicio tardío puedan detenerse espontáneamente (Pavlou \& Gkampeta, 2011).

El papel de una lesión o disfunción cerebral en la aparición de un TA, no está bien esclarecido. Pero en la literatura existe concordancia sobre el papel del hemisferio dominante en los TA, especialmente en la comprensión lectora y en las discapacidades de cálculo (Pavlou \& Gkampeta, 2011)

Los pacientes con Epilepsia del Lóbulo Temporal (ELT) tienen peor rendimiento académico, especialmente en las funciones verbales, cuando el hemisferio cerebral izquierdo está comprometido. Cuando está involucrado el hemisferio 
derecho, hay una discapacidad en las pruebas visuales (Pavlou \& Gkampeta, 2011). Las dificultades de memoria también son características fundamentales de la ELT (Salinas et al., 2018).

Aunque exista variabilidad entre los estudios, se ha observado disfunción de la memoria en los síndromes de epilepsia generalizada, como la epilepsia de ausencia infantil (EAI), así como en epilepsias focales, la Epilepsia Rolándica Benigna (ERB) y Epilepsia del Lóbulo Frontal (ELF) (Salinas et al., 2018).

Los trastornos del aprendizaje pueden ser de dos tipos: TA permanentes o TA dependientes del estado, pero se desconoce la prevalencia de cada uno de ellos. Aunque a menudo no ocurren concomitantemente, los dos tipos de TA pueden ser observables en niños con daño cerebral que tienen convulsiones frecuentes (Pavlou \& Gkampeta, 2011).

En el caso de los trastornos del aprendizaje permanentes, son causadas por lesiones cerebrales y/o disfunción cerebral estable. La causa del daño y/o disfunción cerebral puede ser prenatal, perinatal o posnatal. Muchas anomalías cromosómicas que cursas con encefalopatías no progresivas, están relacionadas con la presencia tanto de epilepsia como de TA permanente. En este caso, ambas son consecuencias distintas de la misma lesión o disfunción cerebral. Por otro lado, la epilepsia puede causar lesiones o disfunciones cerebrales que causan TA permanente (Pavlou \& Gkampeta, 2011).

Por otra parte, los TA dependientes del estado, son potencialmente reversibles y tratables. Son causadas por factores actuales que afectan a los pacientes con crisis epilépticas, como la actividad epiléptica que puede causar TA directamente sin lesión cerebral y/o disfunción y/o el tratamiento antiepiléptico, ambos no necesariamente permanentes (Pavlou \& Gkampeta, 2011)

\section{Metodología}

Esta pesquisa es de tipo cualitativo, donde se utilizo la pesquisa bibliográfica, siguiendo los referenciales de Pereira \& Shitsuka (2018). Para identificar los estudios, la búsqueda se llevó a cabo en las bases de datos bibliográficas LILACS, Medline, Web of Science, SciELO, y PubMed, incluyendo los trabajos publicados desde 2017 hasta la actualidad (febrero de 2021) tanto en español, inglés y portugués. Para la búsqueda se utilizó la combinación de los términos en español "trastornos de aprendizaje en niños con epilepsia", "epilepsia y desarrollo cognitivo", en portugués "transtornos da aprendizagem em crianças com epilepsia", "epilepsia e desenvolvimento cognitivo", y en inglés "learning disorders in children with epilepsy" y "epilepsy and cognitive development. En la fase de tamización, los criterios de inclusión aplicados fueron: inclusión de alguno de los términos de búsqueda en el título, el resumen o palabras claves. Para la fase de elegibilidad, se realizó la lectura de los artículos incluidos y se aplicaron los criterios de exclusión: estudios que no evaluaran los efectos de la epilepsia y en el desarrollo cognitivo y tipos de publicaciones que no incluyeran estudios originales, como revisiones, cartas al editor, capítulos de libro, resúmenes de congresos que no abordan estudios originales y material editorial. Tras la aplicación de los criterios de exclusión, se redujo el número de artículos incluidos para la síntesis cualitativa a 38 .

\section{Resultados y Discusión}

Aún que gran parte de las personas que padecen de epilepsia, pueden llevar una vida igual que las personas que no presentan la enfermedad. Otra gran parte de los casos, aun con limitaciones en la vida cotidiana no tienen afectación en el rendimiento, pero una tercera parte de estos pacientes, pueden tener déficit cognitivo o síntomas neuropsiquiátricos que pueden afectar de forma negativa su calidad de vida (Ninds, 2017).

Kestelman e Kutnowki, 2017, en una pesquisa con 57 niños siendo 24 del grupo de control y 33 del grupo con Epilepsia Benigna de la Infancia (EBI), reportaron diferencias estadísticamente significativas en relación al rendimiento 
escolar entre los dos grupos, mostrando que el grupo de niños epilépticos presentaron bajo rendimiento académico en un 48,5\% de los casos, mientras que el grupo del control sólo presentaron alteraciones en el 8,3\% de los niños.

Los episodios de crisis epilépticas guardan una estrecha relación con la ausencia de los niños en las escuelas y por tanto con el bajo rendimiento académico, por días de clases perdidos. Bazi y col. demostraron que cuanto más frecuente son las convulsiones, mayor es el ausentismo y abandono escolar. Adjunto a esto, también influyen el tratamiento continuo con drogas antiepilépticas con demostrados efectos adversos y el hecho de los padres no querer enviar a los niños para la escuela por temor a la ocurrencia de las crisis (Özkan, Aydin, 2020).

Los modos de intervención sobre los pacientes con epilepsia tienen influencias importantes relacionadas a la cultura y las creencias de los pueblos, afectando la forma como la enfermedad es percibida por las personas y cuáles son las actitudes y políticas que son creadas para combatir este tipo de problema. Constituyendo así otro elemento en detrimento del en los niños que viven con epilepsia (Chew et al., 2017, 2019).

Oostrom et al., 2005, en un reporte de una investigación desarrollada con 42 niños con Epilepsia Idiopática, verificaron que los enfermos tenían un desarrollo inferior al del grupo control y que alrededor del $20 \%$ de los pacientes con epilepsia presentaron dificultades relativas a la escuela.

El estigma sobre la epilepsia es un factor que se incrementa con la falta de información. Para poner un fin o disminuir la discriminación, el repaso de las informaciones sobre el tema es fundamental, desde la identificación de los primeros signos hasta el proceso de abordaje terapéutica. Pero las informaciones sobre los avances en la epilepsia encuentran obstáculos para llegar a los pacientes, familiares y la sociedad, lo que perpetúa el estigma sobre la enfermedad. Es extremamente importante la actuación de los profesionales en la transmisión de informaciones de forma simple y clara para evitar las influencias negativas sobre el proceso docente educativo (Renardin et al., 2019)

Dos estudios discuten sobre factores que al final impactan de forma negativa en el proceso y que pueden comprometer el estado cognitivo en niños con epilepsia, estos factores pueden ser los más diversos, como la etiología de la enfermedad, la frecuencia y gravedad de las crisis epilépticas, la edad de aparición de la primera manifestación patológica. Cabe destacar que estos factores pueden estar presentes de forma aislada o asociados entre sí (Kanner et al., 2020; Marques Rodrigues Lopes, 2020)

Tratamientos con Drogas Antiepilépticas (DAE), pueden influir sobre la cognición tanto positivamente como negativamente. Varios estudios muestran que algunas DAE, pueden estar asociadas a efectos adversos cognitivos, como déficits de aprendizaje y memoria, muchas veces relacionados a la somnolencia diurna producida por los fármacos (Beghi \& Beghi, 2020; Martin et al., 2020; Operto et al., 2020; Taipale et al., 2018). El uso de regímenes de tratamiento con politerapia farmacológica (dos o más DAE simultaneas en dosis terapéuticas) fue una de las causa relacionadas al aumento del déficit cognitivo en niños con epilepsia, cuando se comparó en relación a esquemas de tratamiento en monoterapia (Dale et al., 2019).

La influencia ejercida por el tipo de epilepsia también tiene gran impacto en el desarrollo del aprendizaje del paciente. Estudios indicaron que las crisis epilépticas generalizadas presentaron rangos de inteligencia más bajos que el calculado en los pacientes que tenían crisis epilépticas focales (Bhise et al., 2010; Kim \& Ko, 2016). En las epilepsias focales sintomáticas con origen epileptogénico en el lóbulo frontal, se describieron fundamentalmente déficits de atención y comprometimiento de las funciones ejecutivas (Lagae, 2017).

La influencia del TDAH también ha sido objeto de estudio en de los niños con epilepsia. A pesar de que el TDAH es una comorbilidad del trastorno de aprendizaje, su influencia sobre el desarrollo académico es sumamente importante y se ha descrito alta prevalencia de TDAH en niños con epilepsia. Existen evidencias científicas que apoyan la propuesta que el desarrollo cognitivo y la atención se ven afectados por la actividad epileptogénica propiamente dicha, así como los efectos neurotóxicos e hipnóticos de las medicaciones antiepilépticas, los factores ambientales, emocionales, la extensión de la 
disfunción cerebral adyacente, la edad de inicio de las crisis y factores puramente genéticos. Existe reportes que niños que presentan epilepsia del Lobo temporal presentan un riesgo aumentado de presentar TDAH (Tanquino Peñarreta \& Herdoíza Almeida, 2017; Zambrano et al., 2020).

Autores coinciden en plantear que los pacientes que presentaron epilepsia en edades más tempranas, presentaron mayores probabilidades de problemas cognitivos, posiblemente explicado por la hipótesis que el niño está en una edad critica para la adquisición de las herramientas básicas que sustentan el aprendizaje y las crisis tienden a interrumpir este proceso. Con esto es esperado que presenten déficit cognitivo menor que pacientes que presentaron las crisis de forma más tardía (Ross et al., 2020; Wickens et al., 2017).

Finalmente es bueno destacar que el desempeño escolar en niños con epilepsia es menor que el reportado en la población general que no presentan otros déficits neurológicos, donde la gravedad de la enfermedad y sus características clínicas están parcialmente relacionadas a las implicaciones sociales (Wo et al., 2017).

\section{Conclusión}

Con esta revisión de la literatura podemos concluir que los TA son más frecuentes entre los niños con epilepsia y con predomino significativo en el sexo masculino; la Epilepsia Rolándica es la que se asocia principalmente con los trastornos del aprendizaje; el inicio más temprano de las convulsiones está relacionado con mayor deterioro cognitivo y existe una alta comorbilidad entre el TDAH y los TA.

Factores psicosociales y factores relacionados a la terapéutica farmacológica se han asociado con los déficits de aprendizaje. Los niños mayores tienen una mayor prevalencia de TA, mientras que la falta de apoyo de los padres se ha asociado con mayores dificultades frente a este problema. Por otro lado, el entorno escolar estable y saludable pueden ayudar a mejorar el rendimiento académico y la calidad de vida de los pacientes, a pesar que la gran mayoría de estos niños no tienen acceso a escuelas especializadas para la atención a niños con TA.

\section{Sugerencias para trabajos futuros}

En conjunto, estos resultados pretenden contribuir a una mejor compresión de la relación entre los TA y la epilepsia, así como para el desarrollo de herramientas preventivas y tratamientos eficaces para los sujetos con mayor vulnerabilidad. Así, el trabajo sirve de marco teórico para el inicio de un estudio con niños con TA y epilepsia en escuelas públicas del Paraguay. Consideramos oportuno que en próximas revisiones se incrementen los motores de búsquedas electrónicas y los clasificadores relacionados con el tema, así como ampliar las fronteras de los criterios de inclusión de artículos dentro de las revisiones.

\section{Referencias}

Ahumada, S. P. S., Castro, A. J. E., Barros, R. E. S., Torres, C. M., Cruz, J. C., Guio, J. E. A., et al. (2018). La inclusión: Una base para la educación Vías para la inclusión interdisciplinaria. Ediciones Universidad Simón Bolívar.

Alberrto, H. A. C., Peñarreta, A. M. T. (2017). Course conclusion work (TCC) in Psychiatry. Universidad Central del Ecuador.

Beghi, E., \& Beghi, M. (2020). Epilepsy, antiepileptic drugs and dementia. Curr Opin Neurol, 33 (2), $191-197$.

Beghi, E. (2010). The Epidemiology of Epilepsy. Neuroepidemiology, 54 (2),185-191.

Bhise, V. V., Burack, G. D., \& Mandelbaum, D. E. (2010). Baseline cognition, behavior, and motor skills in children with new-onset, idiopathic epilepsy. Dev Med Child Neurol, 52 (1), 22-26.

Busto, J. E. B. (2018). Trastornos del Lenguaje y Epilepsia. Revista cubana de neurología y neurocirurgía, 8 (2).

Camfield, P., \& Camfield, C. (2015). Prevalence and aetiology of seizures and epilepsy in children. Epileptic Disord, 17 (2), $117-123$.

Chew, J., Carpenter, J., \& Haase, A. M. (2019). Living with epilepsy in adolescence-A qualitative study of young people's experiences in Singapore: Peer 
socialization, autonomy, and self-esteem. Child Care Health Dev, 45 (2), 241-250.

Chew, J., Haase, A. M., \& Carpenter, J. (2017). Individual and family factors associated with self-esteem in young people with epilepsy: A multiple mediation analysis. Epilepsy Behav, 66, 19-26.

Conde Pérez, Y. A., Díaz González, D., Conde Pérez, M., Navia Reyes, I. C., \& Conde Fernández, B. D. (2013). Epidemiología clínica de la epilepsia en la población adulta del municipio Sancti Spíritus. Gaceta médica espirituana, (15), 1608-8921.

Dale, T., Downs, J., Olson, H., Bergin, A. M., Smith, S., \& Leonard, H. (2019). Cannabis for refractory epilepsy in children: A review focusing on CDKL5 Deficiency Disorder. Epilepsy Research. Elsevier B.V., (151), 31-39.

Fiest, K. M., Sauro, K. M., Wiebe, S., Patten, S. B., Kwon, C. S., Dykeman, J., et al. (2017). Prevalence and incidence of epilepsy. Neurology, (88), $296-303$.

Gomes, R. F., Freitas, A. M., Vasques, A. M., Pereira, A. G., Ferreira, E. E. S., \& Wetters Portuguez, M. (2013). Epilepsia de Ausência na Infância e seu Impacto na Aprendizagem. Rev Neurociências, 21 (4), 628-632.

Guillén-Pinto, D., Gonzales, C. V., Vidal, W., Santivañez, C., Vila, J., Juárez, T., et al. (2019). Epilepsia en niños atendidos en el Hospital Nacional Cayetano Heredia de Lima. Rev Neuropsiquiatra, 81 (4), 217.

Kanner, A. M., Helmstaedter, C., Sadat-Hossieny, Z., \& Meador, K. (2020). Cognitive disorders in epilepsy I: Clinical experience, real-world evidence and recommendations. Seizure Seizure: European Journal of Epilepsy, (83), 216-222.

Kestelman, P., \& Kutnowki, C. (2017). Comorbilidad psiquiátrica en niños con epilepsia benigna de la infancia. Med infant, 24 (2), 87-94.

Kim, E., \& Ko, T. (2016). Cognitive impairment in childhood onset epile - psy : up-to-date information about its causes. Korean J Pediatr, 59 (4), $155-164$.

Lagae, L. (2016). The importance of assessing behaviour and cognition in antiepileptic drug trials in children and adolescents. Acta Neurologica Belgica, (171), 425-432.

Marques, K. R. L. (2020). Inclusão escolar de alunos com epilepsia: breve revisão da literatura. Iniciação \& Formação Docente, 7 (3), 652.

Martin, P., Witte, P. A. M, Maurice, T., Gammaitoni, A., Farfel, G., \& Galer, B. (2020). Fenfluramine acts as a positive modulator of sigma-1 receptors. Epilepsy Behav, 105, 106989.

Martinez, E. C. (2018). Epilepsia en la Mujer. Grafisa, S. A. de C. V.

Oostrom, K. J., Teeseling, H. V., Smeets-Schouten, A., Peters, A. C. B., \& Jennekens-Schinkel, A. (2005). Three to four years after diagnosis: Cognition and behaviour in children with "epilepsy only". Brain, (128), 1456-1555.

Operto, F. F., Pastorino, G. M. G., Mazza, R., Carotenuto, M., Roccella, M., Marotta, R., et al. (2020). Effects on executive functions of antiepileptic monotherapy in pediatric age. Epilepsy Behav, 102, 106648.

Özkan, S., \& Aydin, A. (2020). Factors Influencing the School Experience of Children with Epilepsy. Epilepsi, 26 (3), $148-154$.

Pavlou, E., \& Gkampeta, A. (2010). Learning disorders in children with epilepsy. Child's Nervous System, 27, 373-379.

Pereira, A. S., Shitsuka, D. M., Parreira, F. J., \& Shitsuka, R. (2018). Metodologia da pesquisa científica. UFSM. https://repositorio.ufsm.br/bits tream/handle/1/15824/Lic_Computacao_Metodologia-Pesquisa-Cientifica.pdf?sequence=1.

Reilly, C., Atkinson, P., Das, K. B., Chin, R. F. M. C., Aylett, S. E., Burch, V., et al. (2014). Neurobehavioral comorbidities in children with active epilepsy: A population-based study, 133 (6), 1586-1593.

Renardin, D., Soares, L. G., Soares, L. G., Higarashi, I. H., \& Abreu, I. S. (2019). Crianças com epilepsia: percepção e vivência de famílias. Rev Pesqui Cuid é Fundam, 11 (4), 1065-1071.

Ron, A. G. (2017). Epilepsia en Niños y Adolescentes. Esteve.

Ross, E. E., Stoyell, S. M., Kramer, M. A., Berg, A. T., \& Chu, C. J. (2020). The natural history of seizures and neuropsychiatric symptoms in childhood epilepsy with centrotemporal spikes (CECTS). Epilepsy Behav, 103, 106437.

Russ, S. A., Larson, K., \& Halfon, N. (2012). A national profile of childhood epilepsy and seizure disorder. Pediatrics, 129 (2), $256-264$.

Salinas, L., Dalrymple, J., \& MacAllister, W. S. (2018). Memory in children with epilepsy: Utility of the WRAML-2 in generalized and focal epilepsy syndromes. Epilepsy Behav, 89, 30-36.

Smith, A. B., Bajomo, O., \& Pal, D. K. (2015). A meta-analysis of literacy and language in children with rolandic epilepsy. Dev Med Child Neurol, 57 (11), 1019-1026.

Taipale, H., Gomm, W., Broich, K., Maier, W., Tolppanen, A-M., Tanskanen, A., et al. (2018). Use of Antiepileptic Drugs and Dementia Risk-an Analysis of Finnish Health Register and German Health Insurance Data. J Am Geriatr Soc, 66 (6), 1123-1129.

The Epilepsies and Seizures: Hope Through Research. (2020). National Institutes of Health. https://www.ninds.nih.gov/Disorders/Patient-Caregiver Education/Hope-Through-Research/Epilepsies-and-Seizures-Hope-Through.

Wickens, S., Bowden, S. C., \& D’Souza, W. (2017). Cognitive functioning in children with self-limited epilepsy with centrotemporal spikes: A systematic review and meta-analysis. Epilepsia, 58 (10), 1673-1685. 
Research, Society and Development, v. 10, n. 3, e1910313039, 2021

(CC BY 4.0) | ISSN 2525-3409 | DOI: http://dx.doi.org/10.33448/rsd-v10i3.13039

Wo, S. W., Ong, L. C., Low, W. Y., \& Lai, P. S. M. (2017). The impact of epilepsy on academic achievement in children with normal intelligence and without major comorbidities: A systematic review. Epilepsy Research. Elsevier B.V., (136), 35-45.

Zambrano, A., Jair, T., \& Naranjo, K. (2020). Incidencia de la epilepsia del lóbulo temporal: una historia de vida en la escuela. Revista Atlante, (115), 19894155 . 